\section{Cahiers de Narratologie}

Analyse et théorie narratives

10.2 | 2001

La voix narrative

\title{
Les apories de la voix narrative et l'assomption du « je » dans Recuento de Luis Goytisolo (1973)
}

Jacques Soubeyroux

\section{(2) OpenEdition}

1 Journals

Édition électronique

URL : http://journals.openedition.org/narratologie/10296

DOI : 10.4000/narratologie.10296

ISSN : 1765-307X

Éditeur

LIRCES

\section{Édition imprimée}

Date de publication : 1 janvier 2001

Pagination : 455-461

ISBN : 2914561032

ISSN : $0993-8516$

\section{Référence électronique}

Jacques Soubeyroux, «Les apories de la voix narrative et l'assomption du « je » dans Recuento de Luis Goytisolo (1973) », Cahiers de Narratologie [En ligne], 10.2 | 2001, mis en ligne le 01 janvier 2001, consulté le 11 juin 2020. URL : http://journals.openedition.org/narratologie/10296 ; DOI : https:// doi.org/10.4000/narratologie.10296 


\title{
LES APORIES DE LA VOIX NARRATIVE ET L'ASSOMPTION DU « JE » DANS RECUENTO DE LUIS GOYTISOLO (1973)
}

\author{
Jacques SOUBEYROUX \\ Université de Saint-Étienne
}

Le roman que j'ai choisi pour cette communication est le premier tome de la grande tétralogie, intitulée Antagonía et publiée à partir de 1973 par le romancier barcelonais, Luis Goytisolo. Le titre même du roman annonce un inventaire et un récit : "recontar », c'est dénombrer, compter une seconde fois, mais aussi raconter une seconde fois. Le lecteur attend donc le bilan d'une vie, un retour autobiographique sur soi. Or ce qu'il trouve dans ce premier tome, c'est l'autobiographie d'un personnage fictif, qui s'inscrit dans la ligne de ces autobiographies mi-réelles, mi-fictives, ouverte par Pièces d'identité (Señas de identidad, 1966) de Juan Goytisolo, le frère de Luis. Ce premier volume met en place, non seulement les bases référentielles de l'ensemble de l'œuvre, mais aussi sa particulière configuration narrative, caractérisée par une tension permanente entre identité et différence, que j'examinerai en étudiant tour à tour l'articulation entre point de vue et voix narrative, entre récit factuel et discours des personnages, et enfin entre troisième personne et première personne.

\section{1 - Point de vue et voix narrative}

La question du point de vue ne sera pas seulement abordée ici en termes de distance ou de perspective, comme chez Genette, mais aussi en termes de rapports entre énonciation et énoncé, débouchant sur une analyse de la composition liée à la configuration narrative, suivant les propositions de Boris Ouspenski, reprises par Ricœur ${ }^{1}$. J'examinerai trois

1 Paul RICEUR, Temps et récit, t. II, La configuration du temps dans le récit de fiction, Paris, Seuil, 1984, pp. 140-143. 
niveaux du point de vue (temporel, spatial et psychologique), me réservant de revenir plus loin sur les aspects phraséologiques et idéologiques.

$\mathrm{Au}$ plan temporel, le récit suit l'ordre chronologique de l'existence du personnage de Raúl Ferrer, depuis son enfance jusqu'à l'épisode de son emprisonnement à l'âge adulte, les chapitres devenant de plus en plus longs au fur et à mesure que les expériences du personnage deviennent plus complexes : de huit pages pour le chapitre I sur l'enfance à 213 pages pour le chapitre VIII consacré aux relations familiales, sentimentales et politiques de Raúl adulte, le dernier chapitre, de quelque 167 pages, constituant une réflexion sur le «long itinéraire embrouillé » de Raúl Ferrer qui nous a été précédemment raconté. Cet ordre chronologique est pourtant subverti à l'intérieur des chapitres, par une structure en spirale qui détache en tête du chapitre une scène marquante dans la trajectoire du personnage. Ainsi le chapitre VII commence par la libération de Raúl, qui est prolongée par différentes scènes de la conversation qui réunit dans sa maison plusieurs membres de sa famille, et ce n'est qu'ensuite que le récit raconte l'occupation de l'université par les étudiants, l'assaut donné par la police, l'arrestation de Raúl et son interrogatoire, tous événements bien évidemment antérieurs à sa libération. L'anachronie temporelle créée par la structure en spirale ne met pas seulement en relief la scène initiale du chapitre : elle souligne aussi le désordre événementiel qui transcrit la confusion mentale de Raúl et la perte de ses repères identitaires. La composition temporelle du récit est ainsi mise en adéquation avec l'intériorité du personnage.

Au plan spatial, le monde raconté se construit aussi par rapport à la trajectoire de Raúl : nous passons du village de Vallfosca, où sa famille possède une propriété dans laquelle elle s'est réfugiée pendant la guerre civile, au collège religieux, à la caserne, puis à la ville de Barcelone avec ses quartiers populaires et ses monuments, enfin à l'université et à la lutte clandestine contre le franquisme. Cet espace raconté par le narrateur est construit par le regard du personnage, qui évolue avec l'âge. Ainsi, dans le premier chapitre, qui recrée la vie d'une famille de la bourgeoisie catalane le jour de 
Les apories de la voix narrative et l'assomption du « JE » dans Recuento de Luis Goytisolo (1973)

l'entrée des troupes nationalistes dans le village, le regard de l'enfant s'arrête sur des objets ou des événements nouveaux, qui le surprennent : une grenade qui ressemble à une pigne de pin, le défilé des nouvelles recrues avec leur fusil de bois, l'officier caracolant sur son cheval blanc, ou la messe célébrée sur la place du village, suivie de l'hymne franquiste entonné par une foule joyeuse d'hommes portant tous des bérets rouges et des chemises bleues. Plus tard, c'est la Barcelone des quartiers populaires que décrit Raúl adolescent, avec ses bordels et ses rixes entre ivrognes (chapitre IV), puis les musées et les monuments, dans lesquels Raúl étudiant retrouve l'essence de la ville (chapitre VI). L'espace construit par le texte est toujours celui dans lequel évolue ce personnage, comme acteur principal lorsqu'il lance des tracts politiques du haut de la Sagrada Familia, ou comme simple témoin, lorsqu'il écoute une conversation entre membres de sa famille.

Cette fonctionnalité différentielle dont bénéficie le personnage de Raúl s'affirme aussi au plan psychologique, au niveau de la focalisation : à la différence des autres acteurs, qui ne nous sont connus qu'extérieurement, le plus souvent à travers leurs discours directs, Raúl est souvent intériorisé par le narrateur qui rapporte ses souvenirs et analyse ses pensées sous forme de "psycho-récits » débouchant parfois sur de brèves formules de monologue narrativisé.

\section{2 - Récit fictif et récit factuel}

Au plan phraséologique, on est frappé par la fréquence, dès les premières pages du roman, des discours rapportés des personnages, introduits par des verbes déclaratifs, qui émaillent le récit du narrateur. Ces discours, d'abord réduits à une brève réplique, acquièrent progressivement une longueur et une complexité beaucoup plus grandes : discours des religieux du collège, discours patriotiques des militaires à la caserne, mais surtout discours politiques qui viennent s'insérer dans le récit de l'action au cours de réunions, souvent passionnées, des membres de la famille ou d'étudiants amis de Raúl, exprimant les opinions de représentants de toutes les tendances de l'Espagne des années 40-60: monarchistes germanophiles ou anglophiles, phalangistes, bourgeois conservateurs et/ou catalanistes, anciens combattants républi- 
cains ou anarchistes, et surtout étudiants marxistes s'opposant dans d'âpres discussions dont le lecteur, en l'absence d'indications qui auraient dû être données par le narrateur, a parfois du mal à identifier les locuteurs. La confrontation entre ces multiples discours rapportés fait de Recuento un roman authentiquement polyphonique.

Si le narrateur s'efface complètement pour laisser la place aux discours directs des personnages, la fonction de critique de ces discours incombe au personnage de Raúl qui participe à toutes ces discussions, et qui souligne à plusieurs reprises le manque de réalisme de ses camarades, leurs illusions quant au succès d'une hypothétique grève générale qui entraînerait une chute prochaine de la dictature. Ainsi la polyphonie narrative produite par la multiplication des discours rapportés a finalement une portée idéologique : elle sert à dénoncer tout à la fois les contradictions d'un système politique qui aliène totalement les individus et l'inauthenticité de la "langue de bois » de ces discours, qui ne sont qu'un masque derrière lequel ces mêmes individus s'abritent inconsciemment. Cette dénonciation apparaît explicitement dans le chapitre VIII quand le narrateur, prenant le relais des critiques de Raúl, fustige

«le pouvoir d'assignation des mots, leur faculté de stéréotyper la vie quotidienne, de s'interposer entre un être et les choses, entre un être et les autres, entre un être et lui-même. $»^{2}$

La multiplication des discours des personnages est contrebalancée dans le récit par de longues descriptions de la ville de Barcelone et de ses principaux musées et monuments (tout particulièrement l'œuvre majeure de Gaudí, le temple de la «Sagrada Familia»). Ces réflexions historiques ou historico-artistiques, qui relèvent beaucoup plus du récit factuel que du récit fictionnel, viennent augmenter la tension existant au sein du texte entre éléments fictifs et non fictifs. Ce

2 «El poder asignativo de la palabra, su facultad de estereotipar la vida cotidiana, de interponerse entre uno y las cosas, entre uno y los otros, entre uno y sí mismo ". La phrase citée se trouve à la p. 467 de l'édition Madrid, Alianza editorial, 1987, qui sera utilisée tout au long de ce travail. 
Les apories de la voix narrative et l'assomption du « JE »

dans Recuento de Luis Goytisolo (1973)

contenu factuel n'empêche pourtant pas le narrateur de se livrer à des exercices ludiques de transtextualité, consistant à pasticher le style emphatique des guides touristiques lorsqu'il décrit la ville de Barcelone, ou celui des nouvelles pornographiques quand il raconte une scène d'amour entre Raúl et Nuria ${ }^{3}$.

Ces réflexions historico-artistiques du narrateur ne sont pourtant pas de simples excursus, comme on aurait pu le croire a priori, mais une analyse des racines historiques sur lesquelles se fonde l'identité de Raúl, indispensable à l'émergence du Moi du personnage dans le dernier chapitre du roman. Ainsi se dessine une autre convergence entre la trajectoire du personnage et le rôle du narrateur, qui nous conduit à nous interroger sur les relations entre première et troisième personne au sein du récit.

\section{3 - Relations entre première et troisième personne}

Le poète et critique espagnol José Angel Valente a souligné l'absence dans Recuento d'une "voix narrative unificatrice », qualifiant le roman de « texte préalablement narré dont le narrateur n'existe pas encore $»^{4}$. Faut-il considérer la troisième personne narrative dans les huit premiers chapitres de Recuento comme le signe d'une absence de narrateur ${ }^{5}$ ? A la question que pose Genette quand il écrit :

«JE impose mécaniquement et sans aucune échappatoire la relation homodiégétique, c'est-à-dire la certitude que le personnage est le narrateur ; mais IL implique-t-il que le narrateur n'est pas ce personnage?",

je répondrai que, dans Recuento, «IL » n'est qu'un double de «JE », ou plus exactement la figure anonyme d'énonciation qui représente le personnage encore privé d'identité. Si les huit premiers chapitres du roman se caractérisent par l'absence

3 Voir par exemple pp. 147-149 et p. 511.

4 José Angel Valente, "Luis Goytisolo: Recuento », Insula, ${ }^{\circ} 341$, abril 1975, p. 12 : « voz narrativa unificante » et « texto antenarrado cuyo narrador aún no existe ».

5 Sur la possibilité d'un "récit sans narrateu $\mathrm{r}$ » voir Gérard Genette, Nouveau discours du récit, Paris, Seuil, 1983, pp. 66-67 et p. 71 pour la citation suivante. 
de toute référence à la situation d'énonciation, ils n'en contiennent pas moins un certain nombre de signes qui annoncent l'assomption du « JE » dans le chapitre final.

Par exemple le narrateur, apparemment hétérodiégétique, qui assume le récit dans le chapitre I désigne sous le nom de « papa » ou « tante Paquita » les membres de la famille dont il rapporte les discours. Pourtant une phrase au présent, détachée du récit, semble être un discours direct dénué de toute formule introductive : «Cette pigne est une bombe ${ }^{6}$, phrase qu'il faut attribuer au personnage de Raúl enfant, dont on devine la présence tout au long du chapitre, bien que son prénom n'y soit jamais mentionné.

L'émergence du «JE » du narrateur homodiégétique dans le dernier chapitre du roman n'est pas une illumination soudaine du personnage isolé dans sa cellule : elle implique la remontée aux racines historiques, sociales, familiales, nécessaires pour comprendre la construction de l'identité complexe d'un individu nommé Raúl Ferrer Gaminde. Cette reconstruction part de la fondation de Rome et de celle de Barcelone au sein de l'empire romain pour arriver, par tout un enchaînement d'événements historiques, jusqu'à l'apparition de Raúl et à l'affirmation de son existence « hic et nunc »: « moi ici à cet instant ${ }^{7}$.

Pourtant cette identité authentique, qui lui permet enfin de dire «JE », Raúl a conscience de n'avoir pu la conquérir que par l'écriture, seule façon pour lui de

" comprendre le monde à travers lui-même et de se connaître lui-même à travers le monde ${ }^{8}$.

Cette primauté attribuée à l'écriture vient a posteriori éclairer le sens de la configuration narrative du roman : la vie réelle d'un individu n'est qu'un enchaînement fortuit d'événements qui sont en eux-mêmes dénués de toute signification et auxquels seule leur mise en intrigue peut donner un sens. Comme dans Roland Barthes par Roland Barthes, « IL » est

6 «Esta piña es una bomba» (p. 8).

7 « yo aquí en este instante », p. 654.

8 « comprender el mundo a través de sí mismo y conocerse a sí mismo a través del mundo », p. 662. 
dans Recuento le narrateur de la première période pré-identitaire de la vie du sujet, la figure d'énonciation indispensable à la mise en intrigue d'une vie qui ne peut faire sens qu'une fois écrite. La différence, c'est que Recuento n'est pas une autobiographie, même si les différentes étapes de la vie de Raúl Ferrer et son emprisonnement renvoient à des expériences réellement vécues par l'auteur. Les choix narratifs de Luis Goytisolo nous incitent plutôt à parler de "semi-homonymie entre l'expérience réelle (de l'auteur) et l'expérience fictive (du personnage) »9, dans la mesure où l'ultime séquence du dernier chapitre du roman ne signifie aucun retour à la réalité vécue de Luis Goytisolo, mais au contraire l'évasion du personnage-écrivain-narrateur vers l'univers totalement fictif du nouveau roman qu'il entreprend d'écrire. Ce roman, ce sera en fait le tome II d'Antogonía, qui correspond à ce «monde autonome» que le narrateur évoque, qui ne serait pas, comme Recuento, un témoignage sur la réalité, mais une réalité nouvelle qui pourra à son tour servir de référence à son auteur, et pourquoi pas au lecteur, pour créer d'autres œuvres.

La configuration narrative de Recuento est donc en ellemême porteuse de sens. Rejetant toute possibilité de se connaître directement soi-même par une démarche autobiographique, elle signifie que la seule identité qu'il nous soit possible d'atteindre est le miroir que nous offre le récit, non pas de notre propre vie, mais de la vie fictive d'un Autre, d'un double de nous-même. A Rosa Montero qui affirme dans un de ses derniers romans :

«L'identité n'est rien de plus que le récit que nous nous faisons de nous-même »10,

Luis Goytisolo avait répondu par avance en 1983 dans le tome IV d'Antagonía intitulé Théorie de la connaissance:

« Nul ne peut voir que s'il est capable de se voir luimême en train de regarder ce qu'il voit ${ }^{11}$

9 RICEUUR, op. cit. p. 129, note 2.

10 « La identidad no es más que el relato que nos hacemos de nosotros mismos » (La hija del caníbal, 1997).

11 «Sólo ve aquél que es capaz de verse a sí mismo mirando lo que ve », Teoría del conocimiento, Madrid, Alfaguara, 1983, p. 255. 